Revista Destaques Acadêmicos, Lajeado, v. 9, n. 3, 2017. ISSN 2176-3070 DOI: http://dx.doi.org/10.22410/issn.2176-3070.v9i3a2017.1531 www.univates.br/revistas

\title{
O FARMACÊUTICO EM SERVIÇO DE ATENÇÃO SECUNDÁRIA À SAÚDE: ATUAÇÃO EM EQUIPE MULTIPROFISSIONAL PARA PROMOÇÃO DO USO RACIONAL DE MEDICAMENTOS
}

\author{
Alana Lansing ${ }^{1}$, Juliana de Souza ${ }^{2}$, Luciana Carvalho Fernandes ${ }^{3}$, \\ Luís César de Castro ${ }^{4}$, Carla Kauffmann ${ }^{5}$
}

\begin{abstract}
Resumo: Ao longo dos anos, a profissão farmacêutica passou por diversas mudanças. A matriz curricular foi sendo aprimorada conforme a necessidade em formar profissionais preparados para o mercado de trabalho, bem como proporcionar a educação em saúde. A evolução é constante e hoje o profissional graduado é capaz de atuar em diferentes áreas, entre elas, a Atenção Farmacêutica, foco deste estudo que tem como objetivo descrever a experiência de inserção do profissional farmacêutico em equipe multiprofissional em serviço de atenção secundária à saúde, como também suas ações frente ao paciente. $\mathrm{O}$ estudo de caráter transversal foi realizado junto ao Consultório Farmacêutico do Ambulatório de Especialidades Médicas (AEM) da Universidade do Vale do Taquari - Univates. Durante os meses de março a agosto de 2016, foram realizadas 276 consultas farmacêuticas (CF) no AEM, das quais foram compilados os dados referentes a 138 consultas com 98 pacientes atendidos pelas especialidades médicas de cardiologia e pneumologia. A amostra foi constituída por 57 homens $(58,2 \%)$ e 41 mulheres (41,8\%), com idade entre 8 e 92 anos (média de 61,8 anos). Nas CF foram realizadas 227 intervenções no período avaliado, perfazendo uma média de 1,6 serviços por paciente/consulta. Entre os serviços ofertados estão análise do receituário (37,0\%), orientação sobre o acesso ao tratamento farmacológico $(76,1 \%)$, orientação sobre o
\end{abstract}

1 Farmacêutica. E-mail: alana549@hotmail.com

2 Farmacêutica. Centro Clínico Univates, Univates. E-mail: juju_souza@univates.br

3 Farmacêutica. Mestre em Ciências Farmacêuticas. Professora do Centro de Ciências Biológicas e da Saúde, Univates. E-mail: lufernandes@univates.br

4 Farmacêutico. Doutor em Microbiologia Agrícola e do Ambiente. Professor do Centro de Ciências Biológicas e da Saúde, Univates. E-mail: lucamsc@univates.br

5 Farmacêutica. Mestre em Ciências Farmacêuticas. Doutora em Ambiente e Desenvolvimento. Professora do Centro de Ciências Biológicas e da Saúde, Univates. E-mail: carlakauffmann@univates.br 
tratamento medicamentoso (21,7\%) e reconciliação medicamentosa (18,1\%). Assim, é possível verificar-se que a inserção do farmacêutico em equipe multiprofissional pode ser benéfica tanto para a equipe quanto para o paciente, contribuindo para o uso racional da terapia.

Palavras-chave: Atenção farmacêutica. Atenção secundária à saúde. Uso racional de medicamentos.

\section{INTRODUÇÃO}

Ao longo dos anos o profissional farmacêutico passou por altos e baixos em seu modelo de atuação e competências. Nos primórdios dos tempos coloniais foi visto como um curandeiro e entendedor das plantas medicinais para tratamento de pessoas ou animais, porém sem respaldo ou qualquer conhecimento técnico, apenas empírico. Passou por um período em que o profissional era formado em moldes basicamente técnico, mantendo uma relação maior com o produto, o qual pode ser um medicamento ou um material para análise, e dessa forma perdendo-se o cuidado com o paciente, transformando a farmácia em um comércio (BRASIL, 2014; PEREIRA, 2016). Ainda, peregrinou pela fase em farmácias e drogarias, onde o farmacêutico era visto como um dispensador de "caixinhas de medicamentos", afastando-se do perfil de educador em saúde, o qual atualmente está sendo recuperado e praticado (BRASIL, 2014).

A profissão passou por um período complicado, trilhando um caminho solitário, e hoje se encontra em construção de um novo modelo de atuação, com um olhar mais humanizado e voltado ao cuidado, com uma visão ampliada do paciente e suas queixas. Recentemente, nos preparamos para trabalhar em equipes multiprofissionais, colocando o paciente no centro das ações, para que cada profissional possa contribuir com seus saberes (CAMPESE et al., 2016).

Deparamo-nos hoje com uma mudança no estilo e qualidade de vida. Tal variação traz como consequência o aumento da expectativa de vida, levando à inversão da pirâmide demográfica no Brasil, com número significante de idosos (LYRA JÚNIOR et al., 2006; RODRIGUES; OLIVEIRA, 2016). Fato possível devido ao desenvolvimento de novos fármacos e novas metodologias de tratamento, aumentando as chances de cura e sobrevivência. Para acompanhar as necessidades impostas pelo desenvolvimento, muitos métodos de Atenção Farmacêutica são estudados constantemente, a fim de melhorar o atendimento ao público, fornecendo ao farmacêutico, ferramentas para as ações clínicas (CORRER; OTUKI, 2011).

Considerando o cenário atual, este estudo pretende demonstrar em nível secundário de atenção à saúde, a influência de consulta farmacêutica em ambiente multiprofissional na promoção do uso racional da terapia. 


\section{MÉTODOS}

\section{Característica do estudo}

Este estudo transversal possui caráter quantitativo visto que se objetivou avaliar, através de dados coletados em prontuário eletrônico, a interferência da consulta farmacêutica no tratamento de pacientes em atendimento médico especializado. Realizado através do acompanhamento das atividades realizadas pela farmacêutica responsável pelo Ambulatório de Especialidades Médicas, no período de 07 de março a 31 de agosto de 2016, teve embasamento em referências literárias de outros estudos realizados na área de Farmácia Clínica e Atenção Farmacêutica.

\section{Descrição do Ambulatório de Especialidades Médicas (AEM)}

O Ambulatório de Especialidades Médicas está situado na Rua Alberto Muller $\mathrm{n}^{\circ}$ 1151, no campus da Univates, no município de Lajeado - RS, componente do Centro Clínico Univates, o qual abrange outros locais para atuação de profissionais da saúde e estudantes de cursos do Centro de Ciências Biológicas e da Saúde (CCBS), proporcionando atendimento via Sistema Único de Saúde (SUS) à população de Lajeado e municípios vizinhos.

No AEM são ofertados exames e consultas da atenção secundária à saúde. Os pacientes são encaminhados da atenção primária à saúde, quando há necessidade de acompanhamento especializado.

O início das atividades ocorreu no dia 7 de março de 2016, inicialmente com três especialidades médicas: cardiologia, hematologia e pneumologia. O aumento gradual das especialidades médicas está previsto a partir do segundo semestre do mesmo ano até 2019.

A equipe atuante é multiprofissional, sendo que durante o ano de 2016, era composta por enfermeiro, técnicos em enfermagem, médicos especialistas, farmacêutico, fisioterapeuta, assistente social, além de profissionais de outros locais do Centro Clínico Univates, que são adjuvantes, podendo auxiliar no atendimento ao paciente, como biomédico, nutricionista, psicólogo, e educador físico.

A estrutura física conta com salas para realização de exames específicos, suporte para atendimentos de emergência, além de consultórios privativos. Dentre estes, há o Consultório Farmacêutico, onde são realizadas as consultas farmacêuticas.

\section{Descrição do Consultório Farmacêutico (CF)}

Para implantação do Consultório Farmacêutico teve-se como referência literária a legislação que trata das atribuições clínicas do farmacêutico e métodos de Atenção Farmacêutica. 
Em sala privativa, ao lado da farmácia interna, ocorre a consulta farmacêutica. A sala acomoda cadeiras para o paciente e acompanhante, computador e mesa para auxiliar durante a consulta (elaboração de material educativo, análise de receituário, entre outras atividades). Este foi o primeiro consultório do Estado a ser registrado pelo Conselho Regional de Farmácia do Rio Grande do Sul.

As atividades da farmacêutica responsável foram acompanhadas diariamente, no primeiro semestre de 2016, como a consulta farmacêutica que no período ocorria em dois dias da semana para atendimento especializado, além de tarefas na farmácia interna, realizadas nos demais dias, como organização, atendimento de requisições internas para reposição de materiais médico-hospitalares, controle de estoque, compras e tudo que compreende as questões burocráticas, tais como o envio de documentos aos órgãos competentes de fiscalização, elaboração e revisão de Procedimentos Operacionais Padrão (POP), Manual de Boas Práticas, elaboração de material para educação em saúde e protocolos que foram desenvolvidos em conjunto com os outros profissionais, para melhorar adesão ao tratamento e promover a saúde dos pacientes atendidos no AEM, como também a organização de documentos para solicitação de medicamentos do Componente Especializado, quando necessário.

\section{Análise dos serviços farmacêuticos}

A consulta farmacêutica ocorre de forma acolhedora, através de conversa em uma sala individual, garantindo a privacidade e sigilo do paciente. Após atendimento pelo médico especialista, o paciente é encaminhado ao CF. Inicialmente o paciente é recebido e, em seguida, pergunta-se ao mesmo se possui consigo os medicamentos utilizados ou, ainda, se porta as prescrições médicas. Esta ação garante uma melhor orientação e, a partir disso, é possível avaliar se há interação medicamentosa ou problemas relacionados ao uso dos medicamentos.

Em seguida, realiza-se uma anamnese para obter informações sobre o estilo de vida, hábitos e vícios. Na sequência, a prescrição médica é analisada e verifica-se a existência de outras prescrições. Caso o paciente seja polimedicado, ou seja, se há o uso de outros medicamentos e de outros profissionais, é realizada a reconciliação medicamentosa, verificando o uso correto e se há alguma interação entre medicamento-medicamento ou medicamento-alimento, além de sobreposição de medicamentos. Caso seja detectada alguma inconsistência, o médico especialista é contatado para resolução do problema. Se o paciente não for polimedicado, não se faz necessário realizar a reconciliação medicamentosa. Em qualquer caso, no momento da consulta farmacêutica, o paciente recebe orientação sobre sua terapia e outras medidas não-farmacológicas, a fim de otimizar a terapia, melhorar a adesão ao tratamento e dessa forma promover o 
uso racional dos medicamentos. Tais ações geram como consequência, vínculo entre profissional e paciente, além de melhoria na qualidade de vida do mesmo.

Salienta-se que os serviços farmacêuticos são realizados conforme a necessidade do paciente durante a consulta farmacêutica. Cada serviço realizado é registrado no sistema de prontuário eletrônico Tasy ${ }^{\circledR}$. O Tasy ${ }^{\circledR}$ é um sistema de gestão que permite o fluxo de informações entre todos os setores do AEM, unificando processos e informações pertinentes ao paciente em atendimento. Desenvolvido pela Philips ${ }^{\circledR}$, o sistema Tasy ${ }^{\circledR}$ oferece a garantia da confiabilidade das informações, que, uma vez cadastradas, evitam o retrabalho garantindo a segurança e rastreabilidade.

A partir do banco de dados gerado e armazenados no sistema Tasy ${ }^{\circledR}$, foram obtidas as informações necessárias para a realização deste estudo, tais como: idade, sexo, raça, motivo do encaminhamento à consulta, medicamentos utilizados e prescritos na atenção primária a saúde, medicamentos prescritos no AEM, tipo de consulta, serviços farmacêuticos ofertados e diagnósticos.

As informações obtidas foram digitadas em banco desenvolvido no programa Microsoft Excel ${ }^{\oplus}$, no qual foram analisadas posteriormente.

\section{Aspectos éticos}

Cada paciente atendido dentro do AEM assinou um termo autorizando o uso dos dados para pesquisa. Foram selecionados apenas prontuários de pacientes que receberam atendimento médico nas especialidades de cardiologia e pneumologia, utilizando o critério de ordem alfabética para seleção de $50 \%$ dos prontuários analisados. A identidade dos pacientes e médicos foi preservada e mantida em sigilo. Para este estudo, obteve-se a autorização do responsável técnico do AEM.

\section{RESULTADOS E DISCUSSÃO}

Durante os meses de março a agosto de 2016, foram realizadas 276 consultas farmacêuticas com 190 pacientes em atendimento no AEM. Para o presente estudo foram analisados $50 \%$ dos dados registrados, sendo assim, foram compilados os dados referentes a 138 consultas farmacêuticas com 98 pacientes atendidos pelas especialidades médicas de cardiologia, com 62 consultas $(44,9 \%)$, e pneumologia, com 76 consultas $(55,1 \%)$.

Os pacientes amostrados consultaram de uma a quatro vezes no $\mathrm{CF}$ durante o período de estudo, sendo que 69 indivíduos $(70,4 \%)$ passaram por consulta farmacêutica apenas uma vez, 19 (19,4\%) participaram de duas consultas, $9(9,2 \%)$ tiveram três consultas e apenas um utente $(1,0 \%)$ realizou quatro atendimentos. Ainda, é interessante ressaltar que em 33 situações ( $\mathrm{n}=138$ consultas farmacêuticas), os pacientes retornaram ao AEM para atendimento exclusivamente com o farmacêutico, denotando a importância deste profissional. 
A amostra de pacientes atendidos no CF foi constituída por 57 pacientes do sexo masculino $(58,2 \%)$ e 41 do sexo feminino $(41,8 \%)$, distribuídos entre brancos, pardos e negros, com predomínio dos primeiros. A média de idade dos pacientes atendidos foi de 61,8 anos, sendo a idade mínima de 8 anos e a máxima de 92 anos, prevalecendo indivíduos idosos (59,1\%) (Tabela 1).

Entre os motivos de encaminhamento da atenção primária à saúde para atendimento na atenção secundária à saúde prevaleceram doença pulmonar obstrutiva crônica (DPOC) ( $\mathrm{n}=29$ pacientes), hipertensão arterial sistêmica (HAS) ( $\mathrm{n}=22$ pacientes), asma ( $\mathrm{n}=18$ pacientes) e HAS associada à comorbidades ( $n=13$ pacientes).

Tabela 1: Caracterização dos pacientes atendidos no Consultório Farmacêutico do AEM, no período de março a agosto de 2016 (n=98)

\begin{tabular}{l|c|c}
\hline Variável & Frequência (n) & Porcentagem (\%) \\
\hline Sexo & & \\
\hline Feminino & 41 & 41,8 \\
\hline Masculino & 57 & 58,2 \\
\hline Raça & 81 & 82,7 \\
\hline Branca & 6 & 6,1 \\
\hline Negra & 11 & 11,2 \\
\hline Parda & & \\
\hline Faixa etária & 1 & 1,0 \\
\hline menos de 10 anos & 2 & 2,0 \\
\hline 11 a 20 anos & 3 & 3,1 \\
\hline 21 a 30 anos & 7 & 7,1 \\
\hline 31 a 40 anos & 3 & 3,1 \\
\hline 41 a 50 anos & 24 & 24,5 \\
\hline 51 a 60 anos & 28 & 28,6 \\
\hline 61 a 70 anos & 22 & 22,4 \\
\hline 71 a 80 anos & 7 & 7,1 \\
\hline 81 a 90 anos & 1 & 1,0 \\
\hline 91 anos ou mais & & \\
\hline
\end{tabular}

A procura pela especialidade de pneumologia pode ser associada ao estilo e qualidade de vida dos pacientes, assim como a características climatológicas do Vale do Taquari, as quais favorecem problemas respiratórios. Ainda, durante a consulta farmacêutica, muitos pacientes relataram serem 
tabagistas de longa data. Gonçalves et al. (2015) relacionaram sintomas de doenças pulmonares, como dispneia e alterações respiratórias, como fatores que influenciam na qualidade de vida do paciente.

Nas CF são realizados serviços farmacêuticos de acordo com a necessidade de cada paciente, tendo sido realizadas 227 intervenções no período avaliado, perfazendo uma média de 1,6 serviços por paciente/consulta (variação de 1 a 7 serviços por paciente/consulta). Entre os serviços mais frequentemente ofertados estão análise do receituário $(37,0 \%)$, orientação sobre o acesso ao tratamento farmacológico $(76,1 \%)$, orientação sobre o tratamento medicamentoso $(21,7 \%)$ e reconciliação medicamentosa $(18,1 \%)$ (Tabela 2$)$.

Tabela 2: Serviços farmacêuticos ofertados aos pacientes atendidos no Consultório Farmacêutico do AEM, no período de março a agosto de 2016

\begin{tabular}{l|c|c}
\hline Serviço farmacêutico & Frequência (n) & $\begin{array}{c}\text { Porcentagem de serviços } \\
\text { por paciente/consulta (\%) }\end{array}$ \\
\hline Análise do receituário & 51 & 37,0 \\
\hline $\begin{array}{l}\text { Orientação quanto ao acesso da } \\
\text { farmacoterapia }\end{array}$ & 105 & 76,1 \\
\hline Reconciliação medicamentosa & 25 & 18,1 \\
\hline $\begin{array}{l}\text { Orientação sobre o tratamento } \\
\text { medicamentoso }\end{array}$ & 30 & 21,7 \\
\hline $\begin{array}{l}\text { Elaboração de calendário de } \\
\text { administração }\end{array}$ & 11 & 8,0 \\
\hline Outros & 5 & 3,6 \\
\hline Total & $\mathbf{2 2 7}$ & ----- \\
\hline
\end{tabular}

A orientação quanto ao acesso à terapia é fundamental para o paciente compreender o tipo de tratamento e sua procedência. $\mathrm{O}$ modo de aquisição do medicamento era indicado durante a $\mathrm{CF}$, podendo ser adquirido gratuitamente na rede básica de saúde do município ou pelo programa Farmácia Popular do Brasil. Quando o medicamento prescrito pertence ao Componente Especializado da Assistência Farmacêutica, necessitando encaminhamento à Farmácia do Estado, toda organização e gerenciamento do preenchimento de documentos (laudo médico, obtenção de exames necessários comprobatórios e cadastro de paciente, por exemplo) e posterior entrega destes ao paciente é realizado pela farmacêutica do AEM. O paciente também é orientado quando da necessidade de compra do medicamento. A elaboração de material educativo também é considerada como um serviço farmacêutico, entre os quais, um exemplo é a organização de calendário para auxiliar na administração da farmacoterapia, inclusive para pacientes não alfabetizados, onde um sistema de cores é utilizado relacionado ao medicamento (Figura 1 ). 
Figura 1: Calendário utilizado para orientação ao paciente, no Consultório Farmacêutico do AEM

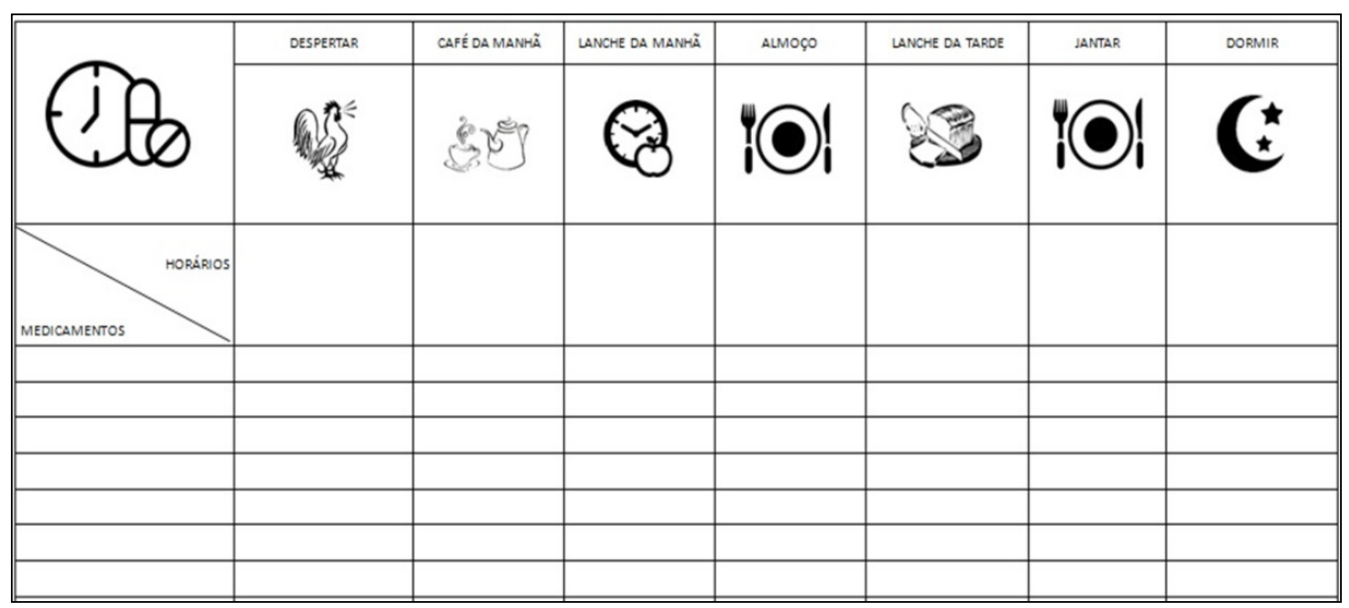

O acompanhamento do paciente por diferentes profissionais é muito benéfico, pois cada profissional é capaz de contribuir com seus saberes para melhorar a qualidade de vida do paciente. No caso do farmacêutico, as ações realizadas em consulta podem identificar problemas relacionados à terapia, administração incorreta, além de uso indevido de medicamentos, seja ele por automedicação ou por prescrições de diferentes médicos. A polimedicação é um risco eminente para saúde, principalmente em idosos, uma vez que pode agravar algumas situações de saúde (FUERTES et al., 2016).

O estudo de Silva et al. (2016) evidencia que o farmacêutico, através de suas ações, é fundamental na redução e controle da HAS, sugerindo inclusive a redução de hospitalizações e mortes prematuras ocorridas pelo uso indevido da farmacoterapia. Contudo, para que a prática clínica seja exercida de forma a beneficiar o paciente com suas ações, é necessário que o profissional desenvolva técnicas de comunicação e habilidades necessárias para construir uma relação de confiança com o paciente (CORRER et al., 2016). 
Tabela 3: Comparativo do número de medicamentos prescritos entre APS e ASS para os pacientes atendidos no Consultório Farmacêutico do AEM, no período de março a agosto de 2016

\begin{tabular}{c|c|c}
\hline $\begin{array}{c}\text { Número de medicamentos } \\
\text { prescritos }\end{array}$ & $\begin{array}{c}\text { Atenção Primária à Saúde } \\
\text { (APS) }\end{array}$ & $\begin{array}{c}\text { Atenção Secundária à } \\
\text { Saúde (ASS) }\end{array}$ \\
\hline 0 & 11 & 1 \\
\hline 1 & 11 & 10 \\
\hline 2 & 11 & 18 \\
\hline 3 & 25 & 30 \\
\hline 4 & 16 & 15 \\
\hline 5 & 15 & 19 \\
\hline 6 & 15 & 13 \\
\hline 7 & 12 & 12 \\
\hline 8 & 7 & 9 \\
\hline 9 & 5 & 0 \\
\hline 10 & 3 & 1 \\
\hline 11 & 1 & 2 \\
\hline 12 & 1 & --- \\
\hline NI & 5 & $\mathbf{4 , 6}$ \\
\hline Média & $\mathbf{4 , 3 ^ { * }}$ & \\
\hline & & 8 \\
\hline
\end{tabular}

${ }^{*} \mathrm{n}=133$

Comparando-se o número médio de medicamentos prescritos por paciente na APS e na ASS (Tabela 3), verifica-se um aumento de 4,3 para 4,6. Contudo, avaliando o número de pacientes polimedicados, isto é, empregando cinco ou mais medicamentos, pode-se verificar que na APS há 59/133 $(44,4 \%)$, enquanto na ASS há 64/138 (46,4\%), ou seja, o aumento é sútil. Ainda, observou-se que em 19 consultas médicas $(13,8 \%)$ houve a redução no número de medicamentos prescritos em relação à APS e em 48 consultas $(34,8 \%)$ não houve alteração no número de medicamentos prescritos, apenas a alteração de classes farmacológicas ou ajuste de doses.

Em 51,4\% das consultas os pacientes tiveram alterações na prescrição, com a inclusão de medicamentos. Porém, por estarem recebendo atendimento na atenção especializada, é provável o diagnóstico de comorbidades relacionadas à queixa principal, através de exames específicos, e, dessa forma, necessária a alteração na farmacoterapia (PINTO et al., 2013). 
Reações adversas e interações medicamentosas podem ser frequentes em polimedicados, além de aumentarem as chances de uso incorreto ou indevido do medicamento (RODRIGUES; OLIVEIRA, 2016). Dessa forma, a presença do profissional farmacêutico junto à equipe multiprofissional e em contato direto com o paciente é fundamental, otimizando a terapia, reduzindo erros de prescrição e de administração da terapia, além de proporcionar educação em saúde.

Entretanto, diferentemente dos estudos realizados por Moraes et al. (2016) e por Marsilio et al. (2016), na presente pesquisa não se observaram incompatibilidades durante as reconciliações farmacêuticas realizadas. Assim como nos estudos supracitados, Lima et al. (2016) também verificaram um alto índice de interações medicamentosas e problemas relacionados aos medicamentos em seu estudo realizado com pacientes idosos.

$\mathrm{O}$ acesso ao medicamento é uma das premissas do uso racional, desta forma, esta informação é repassada aos pacientes que frequentam o CF. Analisando este dado, pode-se observar que a maioria dos utentes foi orientada a buscar nas farmácias da rede básica de saúde do município de Lajeado os seus medicamentos $(25,4 \%)$, seguido de compra por responsabilidade do usuário em farmácias privadas (15,2\%), Farmácia do Estado, uma vez que a terapia faz parte do Componente Especializado da Assistência Farmacêutica (13,8\%) ou acessar através do programa Farmácia Popular do Brasil (11,6\%) (Figura 2).

A perspectiva deste estudo teve grande semelhança ao estudo de Pinto et al. (2013), no qual o resultado foi positivo para interação do farmacêutico com os demais profissionais do ambiente hospitalar, contudo diferenciando-se que no o presente estudo ocorreu em ambulatório de especialidades médicas. Ainda em concordância com Sousa et al. (2016), a partir do presente trabalho se observou que o farmacêutico é essencial para a equipe de saúde na promoção do uso racional de medicamentos, cooperando com médicos especialistas para resolutividade de casos e otimização das terapias e adesão ao tratamento junto dos pacientes. 
Figura 2: Orientação quanto à forma de acesso aos medicamentos prescritos para os pacientes atendidos no Consultório Farmacêutico do AEM, no período de março a agosto de 2016

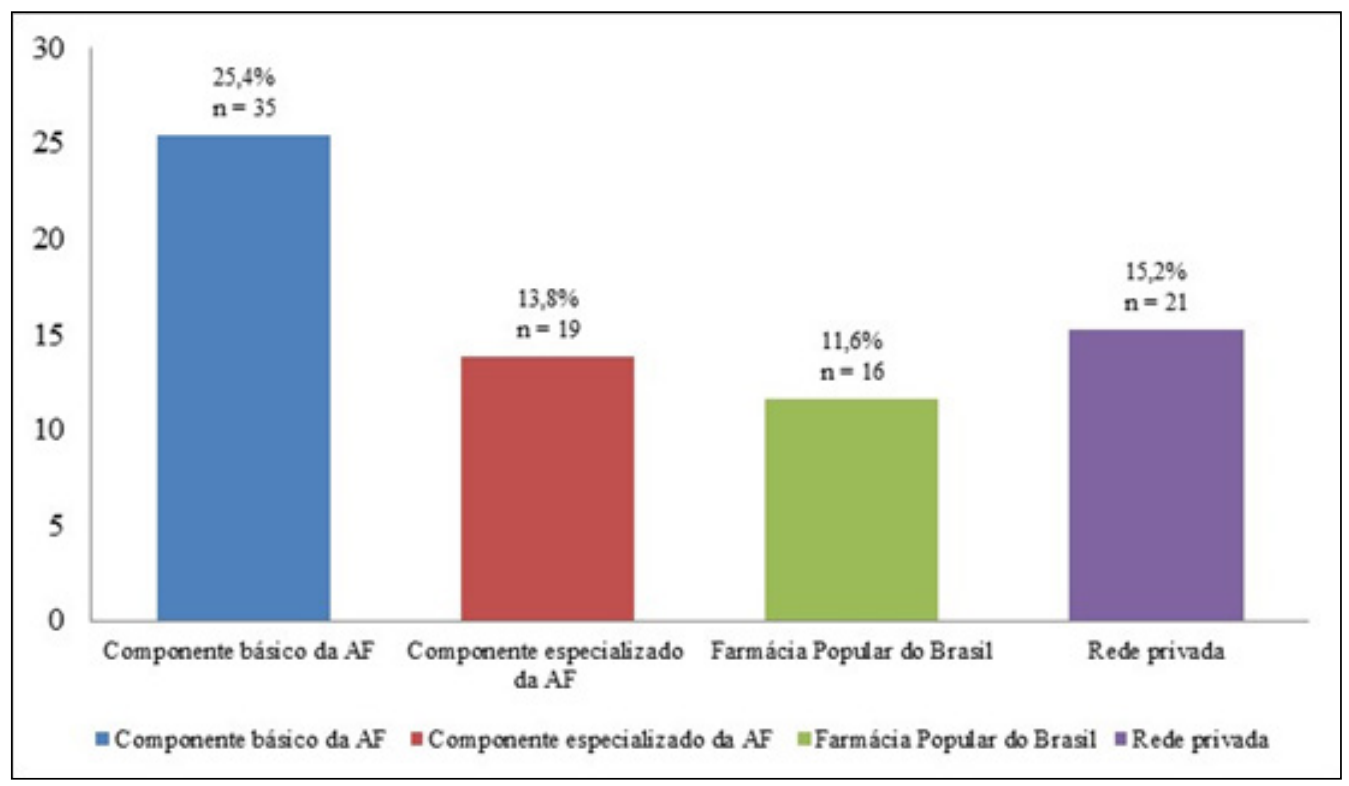

Quanto à implantação do Consultório Farmacêutico no AEM é importante ressaltar que este foi o primeiro do Estado do Rio Grande do Sul a receber o certificado junto ao Conselho Regional de Farmácia. Tal fato demonstra que apesar da existência da legislação (BRASIL, 2013) sobre as atribuições clínicas do farmacêutico para fomentar a prática de orientação e educação em saúde, esta ainda é pouco utilizada, considerando que a mudança no estilo de atuação do profissional vem acontecendo aos poucos, preparando um novo cenário na história da profissão.

Assim como no estudo de Nunes et al. (2008), neste trabalho também foi possível perceber de forma positiva a interferência do farmacêutico no acompanhamento do paciente. Além disso, o farmacêutico teve boa aceitação por parte dos médicos especialistas no AEM, os quais demonstram perceber a importância do farmacêutico para/com o paciente.

\section{CONCLUSÃO}

A análise dos dados obtidos e a possibilidade de acompanhar as atividades do farmacêutico inserido em equipe multiprofissional no AEM, sugerem que a atuação do profissional é benéfica tanto para a equipe quanto para o paciente, podendo reduzir erros de prescrição, problemas relacionados à terapia, administrações incorretas da medicação, as quais podem resultar em danos à saúde. Além de promover a educação em saúde, resultando em 
qualidade de vida e melhores resultados na terapia do paciente. Tais ações, em longo prazo, podem inclusive reduzir o número de reconsultas médicas de pacientes em nível secundário de atenção à saúde.

\section{REFERÊNCIAS}

BRASIL. Conselho Federal de Farmácia. Resolução RDC nº 585, de 29 de agosto de 2013. Regulamenta as atribuiç̃̃es clínicas do farmacêutico e dá outras providências. Diário Oficial da União, 25 de agosto de 2013. Seção 1.186 p.

BRASIL. Ministério da Saúde. Cuidado farmacêutico na atenção básica. Caderno 2: capacitação para implantação dos serviços de clínica farmacêutica. Brasília: Ministério da Saúde, 2014. 308 p.

CAMPESE, M.; SOARES, L.; LEITE, S. N.; FARIAS, M. R. O devir da profissão farmacêutica e a clínica farmacêutica. In: SOARES, L.; FARIAS, M. R.; LEITE, S. N.; CAMPESE, M.; MANZINI, F. Assistência Farmacêutica no Brasil - política, gestão e clínica. Atuação clínica do farmacêutico - Volume V. Santa Catarina: Editora UFSC, 2016. p. 21-43.

CORRER, C. J.; NOBLAT, L. A. C. B.; CASTRO, M. S. Modelos de seguimento farmacoterapêutico. .In: SOARES, L.; FARIAS, M. R.; LEITE, S. N.; CAMPESE, M.; MANZINI, F. Assistência Farmacêutica no Brasil - política, gestão e clínica. Atuação clínica do farmacêutico - Volume V. Santa Catarina: Editora UFSC, 2016. p.221-251.

CORRER, C. J.; OTUKI, M. F. Método clínico de atenção farmacêutica. Março de 2011. Disponível em: <http://www.saude.sp.gov.br/resources/ipgg/assistenciafarmaceutica/otuki-metodoclinicoparaatencaofarmaceutica.pdf $>$. Acesso em: 12 set. 2016.

FUERTES, D. G.; FERNÁNDEZ, E. V.; CRESPÍN, M. QT Longo e Torsades de Pointes Induzidos por Fármacos em Pacientes Idosos Polimedicados. Arq. Bras. Cardiol., v. 106, n. 2, p. 156-59, 2016.

GONÇALVES, B. F. T.; MELLO, F. M.; COSTA, C. C.; PEREIRA, M. B.; MANCOPES, R. Qualidade de vida em voz na doença pulmonar crônica. Revista CEFAC, v. 17, n. 6, p. 1773-80, 2015.

LIMA, T. A. M.; FAZAN, E. R.; PEREIRA, L. L.V.; GODOY, M. F. Acompanhamento farmacoterapêutico em idosos. Arq. Ciênc. Saúde, v. 23, n. 1, p. 52-57, 2016.

LYRA JÚNIOR, D.P.; AMARAL, R. T.; VEIGA, E. V.; CÁRNIO, E.C.; NOGUEIRA, M.S.; PELÁ, I. R. A farmacoterapia no idoso: revisão sobre a abordagem multiprofissional no controle da hipertensão arterial sistêmica. Revista LatinoAmericana de Enfermagem; v. 14, n. 3, p. 435-41, 2006. 
MARSILIO, N. R.; SILVA, D.; BUENO, D. Incompatibilidades medicamentosas em centro de tratamento intensivo adulto de um hospital universitário. Rev. Bras. Ter. Intensiva, v. 28, n. 2, p. 147-153, 2016.

MORAES, G. G.; ROSA, K.; FRANTZ, M. R.; BATISTA, M. S.; SCHNEIDER, A. P. H. Atuação do farmacêutico residente em uma unidade de pronto atendimento: contribuindo para a promoção da saúde. Revista de Epidemiologia e Controle de Infecção, v. 6, n. 4, p. 181-184, 2016.

NUNES, P. H. C.; PEREIRA, B. M. G.; NOMINATO, J. C. S.; ALBUQUERQUE, E. M.; SILVA, L. F. N.; CASTRO, I. R. S.; CASTILHO, S. R. Intervenção farmacêutica e prevenção de eventos adversos. Revista Brasileira de Ciências Farmacêuticas, v. 44, n. 4, p. 691-99, 2008.

PEREIRA, L. R. L. Da botica a clínica farmacêutica. In: SOARES, L.; FARIAS, M. R.; LEITE, S. N.; CAMPESE, M.; MANZINI, F. Assistência Farmacêutica no Brasil - política, gestão e clínica. Atuação clínica do farmacêutico - Volume V. Santa Catarina: Editora UFSC, 2016. p. 91-113.

PINTO, I. V. L.; CASTRO, M. S.; REIS, A. M. M. Descrição da atuação do farmacêutico em equipe multiprofissional com ênfase no cuidado ao idoso hospitalizado. Revista Brasileira de Geriatria e Gerontologia, v. 16, n. 4, p. 747-58, 2013.

RODRIGUES, M. C. S.; OLIVEIRA, C. Interações medicamentosas e reações adversas a medicamentos em polifarmácia em idosos: uma revisão integrativa. Revista LatinoAmericana de Enfermagem; v. 24, n. e2800, p. 1-17, 2016.

SILVA, L. G. A.; ARAGÃO, C. C. V.; SABINO, W. Pressão arterial e atenção farmacêutica: o cuidado faz a diferença. Rev. Aten. Saúde, v. 14, n. 47, p. 12-18, 2016.

SOUSA, I. F.; BASTOS, P. R. H. O. Interdisciplinaridade e formação na área de Farmácia. Trab. Educ. Saúde, v. 14, n. 1, p. 97-117, 2016. 